CHEMICAL SOCIETY REVIEWS (ISSN: 0306-0012) 43: (14) pp. 4940-4952. (2014) DOI: $10.1039 /$ C3CS60469A

Received 20 Dec 2013, First published online 20 Mar 2014 


\title{
Challenges in Modelling Homogeneous Catalysis: New Answers from Ab Initio Molecular Dynamics to the Controversy over the Wacker Process
}

\author{
András Stirling, ${ }^{* a}$ Nisanth N. Nair, ${ }^{* b}$ Agustí Lledós ${ }^{* c}$ and Gregori Ujaque ${ }^{* c}$ \\ Received (in $X X X, X X X) X$ th $X X X X X X X X X 20 X X$, Accepted Xth $X X X X X X X X X 20 X X$ \\ ${ }_{5}$ DOI: 10.1039/b000000x
}

We present here a review on the mechanistic studies of the Wacker process stressing the long controversy about the key reaction steps. We give an overview of the previous experimental and theoretical works on the topic. Then we describe the importance of the most recent Ab Initio Molecular Dynamics (AIMD) calculations in modelling organometallic reactivity in water. As a prototypical example of homogeneous 10 catalytic reactions, the Wacker process poses serious challenges to modelling. The adequate description of the multiple role of the water solvent is very difficult by using static quantum chemical approaches including cluster and continuum solvent models. In contrast, such reaction systems are suitable for AIMD, and by combining with rare event sampling techniques, the method provides reaction mechanisms and the corresponding free energy profiles. The review also highlights how AIMD has helped to obtain

15 novel understanding of the mechanism and kinetics of the Wacker process.

\section{Introduction}

The Wacker process is an outstanding example of homogeneous catalytic reaction applied in industry. ${ }^{1,2,3}$ The process was 20 discovered in the late 1950 s by Schmid et al. at Wacker Chemie for producing acetaldehyde from ethene and oxygen (Scheme 1). It allowed the use of ethene, instead of the more expensive and energy-rich acetylene as feedstock for aliphatic organic chemistry. Moreover, this reaction was the starting point for the 25 development of catalytic palladium chemistry.

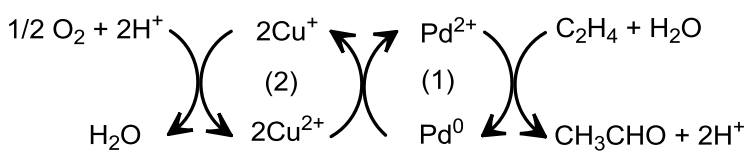

Scheme 1 General scheme of the Wacker process. Numbers correspond to chemical equations.

The stoichiometric oxidation of ethene by $\mathrm{Pd}(\mathrm{II})$ salts was 30 known for a long time, but the crucial discovery of the Wacker process was that the catalyst could be regenerated (oxidized) by molecular oxygen in the presence of the cocatalyst $\mathrm{CuCl}_{2}$. (eq. 13).

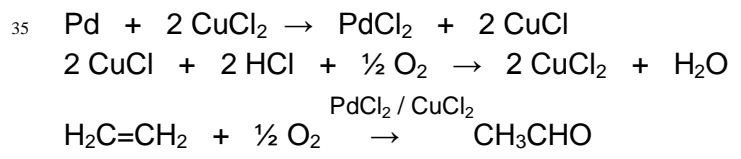

40 The publication of the Wacker process focused the research of many groups on the chemistry of $\mathrm{Pd}(\mathrm{II})$ thus developing new reactions for the addition of several nucleophiles to alkenes. Importantly, the formation of new $\mathrm{C}-\mathrm{O}, \mathrm{C}-\mathrm{N}$ and $\mathrm{C}-\mathrm{C}$ bonds, including intra- and intermolecular reactions has been extensively 45 investigated. $^{4}$ The application of this process in synthesis, however, required development of appropriate conditions, such as those worked out by Tsuji. ${ }^{5}$ A common issue in designing an economic catalytic cycle for these olefin oxidations is the efficient regeneration of the active $\mathrm{Pd}(\mathrm{II})$ catalyst. In this sense, 50 recent progress is achieved moving toward using molecular oxygen as oxidant in synthetic reactions. ${ }^{6}$

The rate expression for the Wacker process was established by Henry's group in a seminal work, exhibiting a first order in ethene and the catalyst (taking $\left[\mathrm{PdCl}_{4}\right]^{2-}$ as the resting state) and ${ }_{55}$ inhibition by $\left[\mathrm{H}^{+}\right]$and $\left[\mathrm{Cl}^{-}\right]$to the second order (eq. 4). ${ }^{7}$ Additional kinetic studies by the same group also established a new rate equation at high $\left[\mathrm{Cl}^{-}\right]$with no proton inhibition term (eq. $5)^{8}$

$$
\text { Low }\left[\mathrm{Cl}^{-}\right] \Rightarrow v=k \frac{\left[\mathrm{PdCl}_{4}^{2-}\right]\left[\mathrm{C}_{2} \mathrm{H}_{4}\right]}{\left[\mathrm{H}_{3} \mathrm{O}^{+}\right]\left[\mathrm{Cl}^{-}\right]^{2}}
$$

60

$$
\text { High }\left[\mathrm{Cl}^{-}\right] \Rightarrow v=k \frac{\left[\mathrm{PdCl}_{4}^{2-}\right]\left[\mathrm{C}_{2} \mathrm{H}_{4}\right]}{\left[\mathrm{Cl}^{-}\right]}
$$

Since its discovery a lot of experimental and theoretical studies have been carried out in order to elucidate the reaction 65 mechanism for this process. The reaction goes through the net addition of a $\mathrm{Pd}$ and a hydroxide group across the $\mathrm{C}=\mathrm{C}$ bond, 
named "hydroxypalladation". How this process takes place is crucial and motivated the controversy on the mechanism over decades.

The first part of the review briefly summarizes the controvery 5 over the Wacker mechanism arising from experimental mechanistic studies. The second and most extended part summarizes some of the most important challenges theoreticians face when studying the Wacker process. The review of the computational studies, from the early molecular orbital analysis 10 to the most recent AIMD simulations, also shows the debate.

\section{Mechanistic studies from experiment}

Two mechanisms have been postulated for the explanation of the rate law (Scheme 2): a) anti nucleophilic attack by a water molecule from the bulk prior to the rate determining step of the 15 process, b) syn nucleophilic attack by a coordinated hydroxide group located cis to the alkene as rate determining step. They are also described by cis or trans terms, or by inner- or outer-sphere mechanisms in the literature. This nucleophilic addition is intimately linked to the proton inhibition observed in the rate law 20 and has been at the core of the mechanistic debate.

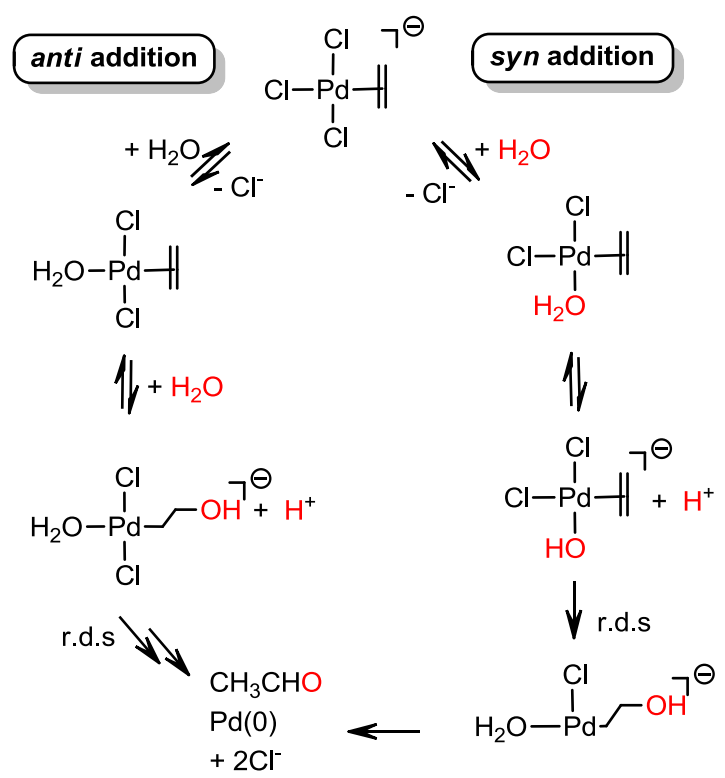

Scheme 2. The two postulated reaction pathways for the Wacker process.

The mechanistic analyses in the laboratory have been mainly done by means of deuterium-labeling experiments and 25 stereochemical studies.

The oxidation of $\mathrm{C}_{2} \mathrm{H}_{4}$ carried out in $\mathrm{D}_{2} \mathrm{O}$ gives $\mathrm{CH}_{3} \mathrm{CHO}$ as the only product, ${ }^{9}$ whereas the process using $\mathrm{C}_{2} \mathrm{D}_{4}$ in $\mathrm{H}_{2} \mathrm{O}$ generates $\mathrm{CD}_{3} \mathrm{CDO} .7$ These results indicate that hydrogen transfers between carbon atoms take place intramolecularly (there 30 is no exchange with protons from the solvent). The measured kinetic isotope effect was found to be small $\left(\mathrm{k}_{\mathrm{H}} / \mathrm{k}_{\mathrm{D}}=1.07\right)$, consistent with the fact that the hydrogen transfer process is not involved in the rate determining step. A comparative isotope effect was evaluated for the oxidation of 1,2-dideuterioethene ${ }_{35}\left(\mathrm{C}_{2} \mathrm{H}_{2} \mathrm{D}_{2}\right) ;{ }^{10}$ the hydroxypalladate intermediate [ $\mathrm{L}_{\mathrm{n}} \mathrm{Pd}(\mathrm{CHD}-$ $\mathrm{CHDOH})$ ] evolves to give two different products: $\mathrm{CHD}_{2} \mathrm{CHO}$ (D shift) and $\mathrm{CH}_{2} \mathrm{DCDO}$ (H shift), see Scheme 3. The product ratio measures the isotope effect. The obtained kinetic ratio (D shift / $\mathrm{H}$ shift) of 1.70 was taken as a support for the previous 40 hydroxypalladation step as rate-determining step. ${ }^{10}$ This assertion was later supported by means of analyzing the correlation between relative rates of oxidation to ionization potentials, HOMOs and LUMOs. ${ }^{11}$

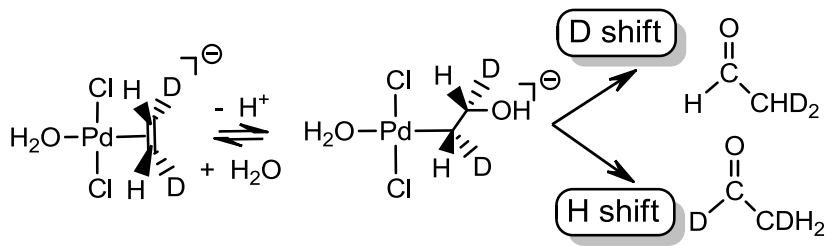

${ }_{45}$ Scheme 3 Comparative isotope effect for the oxidation of 1,2dideuterioethene.

The stereochemical studies on the Wacker mechanism carried out by Stille and co-workers supported an anti addition (in high $\left.\left[\mathrm{Cl}^{-}\right]\right)$. These authors showed that uncoordinated nucleophile ${ }_{50}$ attacks at the most substituted carbon atom of the double bond; this was the exclusive reaction observed for the olefins studied (cyclic, linear, etc). ${ }^{12}$ Additional stereochemical studies by other groups also supported the anti addition for the Wacker process. $^{13,14}$

55 Bäckvall, Akermark and co-workers ${ }^{15}$ used dideuterio-ethene (cis and trans species) with an excess of $\mathrm{LiCl}$ (which forms 2chloroethanol along with acetaldehyde) to carry out their mechanistic studies. The stereochemistry of hydroxypalladation for the reaction pathway leading to 2-chloroethanol 60 unequivocally showed that the process takes place through an anti mechanism. They proposed that the trans hydroxypalladation is an equilibrium process prior the rate determining step; the subsequent reaction step corresponding to the loss of chloride is the rate determining step (Scheme 4). Such mechanistic proposal 65 obeys the rate law. This contribution was crucial for supporting the anti pathway, and nowadays there is a general consensus that anti pathway is the operative one (at least for high $\left[\mathrm{Cl}^{-}\right]$, see below).

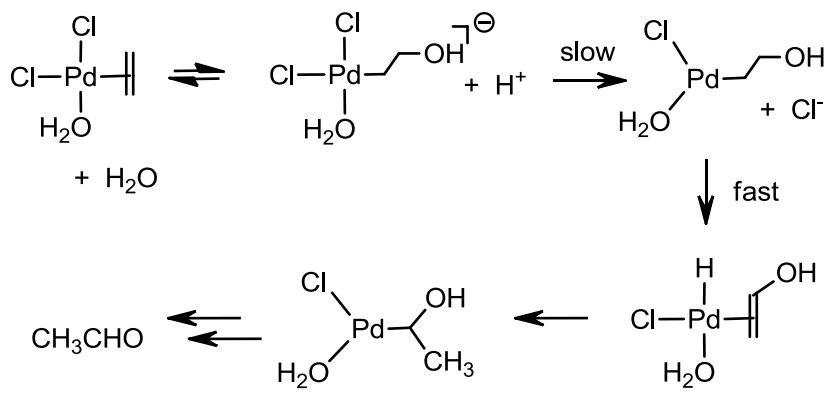

70 Scheme 4 Main steps of the reaction mechanism proposed by Bäckvall and cowokers. ${ }^{15}$

Some authors, however, suggested that these stereochemical studies supporting the anti pathway might not be relevant to the mechanism of the Wacker process because the reaction 75 conditions are not those of the common Wacker process (low $\left[\mathrm{Cl}^{-}\right.$ ]). $3^{, 16}$ The main argument is that the nature of the nucleophilic attack, whether it is an equilibrium process or not, must be crucial to differentiating between the two pathways.

Kinetic experiments on the oxidation of ethene by 
$\left.\left[\mathrm{PdCl}_{3} \text { (pyridine) }\right)^{-}\right]$in aqueous solution (in the presence and absence of $\mathrm{CuCl}_{2}$ ) showed that the rate law was the same at low $\left[\mathrm{Cl}^{-}\right]$as for the regular catalyst used in the Wacker process; the reaction, however, was significantly slower. ${ }^{17}$ The product 5 distribution observed was consistent with a mechanism involving an anti addition at high $\left[\mathrm{Cl}^{-}\right]$and a syn addition at low $\left[\mathrm{Cl}^{-}\right]$. These conclusions brought the community to the consensus that the anti pathway is the operative one at high $\left[\mathrm{Cl}^{-}\right]$concentration.

Henry's group also performed stereochemical investigations 10 by measuring the rates of isomerisation and oxidation of a set of isotopically substituted olefins. For the case of $\left[\mathrm{D}_{2}\right]$ allylic alcohol, ${ }^{18}$ this species is capable of showing isotopic scrambling. Thus, such studies should assess whether nucleophilic attack is an equilibrium process. Although quinone was used as reoxidant for ${ }_{15} \mathrm{Pd}$, the rate law observed was the same as in the Wacker process (eq. 4). ${ }^{18}$ The isomerisation between $\mathrm{H}_{2} \mathrm{C}=\mathrm{CHCD}_{2} \mathrm{OH}$ and $\mathrm{D}_{2} \mathrm{C}=\mathrm{CHCH}_{2} \mathrm{OH}$ was indeed not observed (Scheme 5). The lack of isotopic scrambling indicated that the nucleophilic addition is not an equilibrium process. This observation was shown as an 20 evidence against the anti mechanism, although this result did not confirm the syn pathway neither.

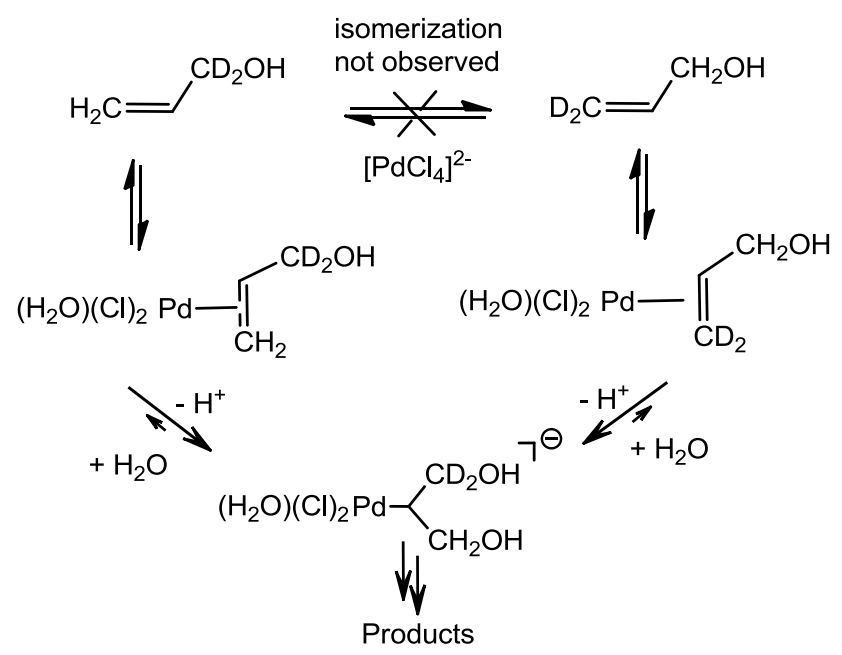

Scheme 5 Mechanistic proposal for $\left[\mathrm{D}_{2}\right]$ allyl alcohol isomerisation, though such process was not observed.

25 With the purpose to scrutinize the stereochemistry of the Wacker products under low $\left[\mathrm{Cl}^{-}\right]$and $\left[\mathrm{CuCl}_{2}\right]$, Henry devised some chirality transfer experiments. ${ }^{19}$ The isomerisation of 2$\left[\mathrm{D}_{3}\right]$ Methyl-4-metyl-1,1,1,5,5,5-hexafluoro-3-penten-2-ol was investigated at low $\left[\mathrm{Cl}^{-}\right]$. The rate law was the same as for the

${ }_{30}$ Wacker process (eq. 4) whereas the stereochemistry of the product was consistent with syn addition. The isomerization of the same chiral reactant at high $\left[\mathrm{Cl}^{-}\right]$was also evaluated. ${ }^{20}$ The rate law was also consistent with that of the Wacker process at high $\left[\mathrm{Cl}^{-}\right]$(eq. 5) but the obtained product was consistent with the 35 anti pathway. Thus, consensus was reached for the process at high $\left[\mathrm{Cl}^{-}\right]$but the controversy is still continuing for the reaction pathway at low $\left[\mathrm{Cl}^{-}\right]$. Additional investigations were performed by carefully selecting chiral allylic alcohols $\left(\mathrm{R}^{1} \mathrm{C}_{\mathrm{a}} \mathrm{HOH}-\right.$ $\mathrm{C}_{\mathrm{b}} \mathrm{H}=\mathrm{C}_{\mathrm{c}} \mathrm{HR}^{2}$ ) to obtain chiral products (with stereocenter $\mathrm{C}_{\mathrm{c}}$ ) 40 providing stereochemical evidence of the nucleophilic attack. ${ }^{21}$ The results supported an anti addition at high $\left[\mathrm{Cl}^{-}\right]$and a syn addition at low $\left[\mathrm{Cl}^{-}\right]$.
Note that one factor that is usually not considered in most mechanistic analyses is related to the role of $\mathrm{CuCl}_{2}$. The validity 45 of this assumption has been called into question by Hosokawa and co-workers ${ }^{22}$ (proving the activity of a $\mathrm{Pd}-\mathrm{Cu}$ heterometallic as a catalyst for the process) and by Sigman and co-workers on a modified version of the Pd catalyst (that works without $\mathrm{CuCl}_{2}$ ). ${ }^{23}$

Overall, the extensive kinetic and stereochemical studies on

50 the Wacker process highlight the high sensitivity of the reaction to the substrate, the catalyst and the reaction conditions. Theoretical calculations on the mechanism also reflected the difficulty to understand it at a molecular level, and in this case, even more importantly they highlighted the complications to 55 reproduce free energy differences between the steps involved.

\section{Mechanistic studies from theory}

This section is divided in several subsections. The first gives an overview of the theoretical modelling in mechanistic studies and focuses on cluster calculations. Then we critically survey the 60 static quantum chemical calculations on the Wacker mechanism and point out how systematic improvements in the models led to more reliable conclusions. Then in the next subsection we summarize how and why dynamics effects can remarkably improve the reliability of the models. Finally we summarize 65 recent ab initio molecular dynamics calculations performed on the Wacker process and their conclusions.

\section{Reaction mechanisms from computation}

Obtaining the reaction mechanism of a chemical reaction in a complex system by experiments is still a challenging problem 70 (see above). Often, electronic structure methods like the density functional theory (DFT) combined with powerful optimization methods are found to be very useful in predicting the reaction mechanism and kinetics of chemical reactions. Computational approaches are generally based on computing Gibbs energy 75 differences between reactants, products and transition states, or along a (pre-defined/automatically traced) reaction coordinate. Analysis of molecular structures along a reaction pathway provides deep insights into the mechanism of a reaction and computed free energy barriers can be linked to experimentally 80 measurable kinetic parameters.

Exploration of reaction mechanisms requires not only an accurate electronic structure description but also information about the atomic motions. The atomic motions are determined by the underlying potential energy surface (PES) given by the Born${ }_{85}$ Oppenheimer approximation (BOA) as

$$
E\left(\mathbf{R}_{I}\right)=E_{n n}\left(\mathbf{R}_{I}\right)+\left\langle\Psi_{0}\left|\widehat{H}_{e}\left(\mathbf{R}_{I}, \mathbf{r}_{i}\right)\right| \Psi_{0}\right\rangle
$$

where $E\left(\mathbf{R}_{I}\right)$ is the PES of the system as a function of the nuclear 90 coordinates $\mathbf{R}_{I} ; E_{n n}$ is the Coulomb interaction for the nuclei; $\Psi=\Psi_{0}\left(\mathbf{r}_{\mathrm{i}}\right)$ is the ground state electronic wavefunction at nuclear configuration $\mathbf{R}_{I}$ and $\mathbf{r}_{\mathrm{i}}$ is the coordinate vector of the electrons. ${ }^{24}$ The Hamiltonian $\widehat{H}_{e}\left(\mathbf{R}_{I}, \mathbf{r}_{i}\right)$ is given by

$$
\widehat{H}_{e}\left(\mathbf{R}_{I}, \mathbf{r}_{i}\right)=-\sum_{i} \frac{\hbar^{2}}{2 m_{e}} \nabla_{i}^{2}+V_{e e}\left(\mathbf{r}_{i}\right)+V_{n e}\left(\mathbf{R}_{I}, \mathbf{r}_{i}\right)
$$

95 and it depends only parametrically on the classical nuclear positions $\mathbf{R}_{I}$. The PES, that can be found by various methods, is 
assumed to contain all the necessary information to describe a chemical reaction. ${ }^{25}$ In most cases this assumption is correct. Typical exceptions are non-adiabatic reactions where the BOA fails and surface crossings have to be accounted for.

${ }_{5}$ Regarding the Wacker process, since the catalyst has a late transition metal and the reactions contain proton/charge transfer intermediates, the underlying electronic structure method should account for the chemical reactivity of transition metal complexes and charge transfer states. DFT based computations are widely 10 used for studying transition metal chemistry. The exchangecorrelation functional should be carefully chosen to adequately describe static correlation effects and dispersion interactions. Moreover, the relativistic effects may also become crucial to obtain correct reactivity and energetics. All these factors can 15 result in significant errors in the prediction of structures and energies. ${ }^{26}$ Modern theoretical calculations of the Wacker process (reported in the past one decade) were using Generalized Gradient Approximation (GGA) and hybrid density functionals. Many of them are shown to behave properly compared to high 20 level ab initio methods for the bond activation by $\mathrm{Pd}$ complexes. $^{27}$

When the reactive system is composed of a few atoms, a reaction mechanism is understood as a motion which takes the system from the reactant minimum to the product minimum by going 25 through the transition state (TS) along the minimal energy path on the PES. The minima can be located on the PES by efficient optimization techniques. ${ }^{25}$ The TS can be identified on the basis of the fact that it is a first order saddle point. ${ }^{24}$ While these calculations imply zero $\mathrm{K}$ temperature and no atomic 30 fluctuations, dynamic information can be obtained implicitly by invoking the Transition State Theory (TST) approach. ${ }^{28}$

$$
k_{T S T}=\frac{k T}{h} \exp \left(\frac{-\Delta G^{\#}}{k T}\right)
$$

where $k_{T S T}$ is the rate constants from the TST model, and $\Delta G^{\#}$ is the activation Gibbs energy necessary to bring the reactant 35 molecules to the activated state. The entropy contributions to $\Delta G^{\#}$ are usually estimated with the Ideal Gas - Rigid Rotor - Harmonic Oscillator approximations for the translational, rotational and vibrational degrees of freedom, respectively. ${ }^{24}$ If necessary, additional Intrinsic Reaction coordinate (IRC) calculations can 40 refine the reaction path connecting reactants and products with the TS. The reactive motions can be further analysed along the $\mathrm{IRC}^{29}$

As the complexity of the system increases, computational approaches also face severe limitations in predicting reaction 45 mechanisms correctly. Primarily, results are dependent on the molecular models employed in the calculation; deviations from experimental conditions can result in wrong prediction of mechanism and kinetics. Secondly, with the increase in the system complexity, several possible reaction pathways have to be 50 analysed to identify the reaction mechanism and this procedure often requires inputs from our chemical intuitions.

These two aspects have crucial relevance for the Wacker process where the catalyst precursor is undergoing multiple ligand exchange reactions in solution and solvent (water) 55 molecules are playing a direct role in the reactions. Solvent effects are crucial here, as both solute and solvent molecules are polar. One of the ways to incorporate solvent effects in quantum chemical computations is by employing implicit solvent models, where the solute and the solvent interactions are included by a 60 polarizable continuum model (PCM) ${ }^{30}$ Certainly this approach represents a gross simplification of the reactive system and can be suitable when the following criteria are met: the reaction is localized onto a region of a few atoms; the interaction between solute and solvent are not dominated by H-bonding; the solvent 65 does not participate in the reaction. Clearly, when any of these criteria is not fulfilled, the description of the reaction misses an essential ingredient of the mechanism and contributions to the reaction free energy arising from the presence of solvent may be significantly underestimated. The Wacker process is a prototype 70 of such a situation. The two-fold role of the water solvent (reactant and solvent) introduces significant challenges for modeling. From the beginning it has been realized in the gasphase cluster calculations, that the reactivity of a water molecule is influenced by its direct interaction with neighbouring solvent 75 water molecules. Thus most of the static quantum chemical calculations of the reaction were using the PCM model together with a few (explicit) water molecules partially solvating the Pdcomplex.

\section{Static calculations on the Wacker process}

${ }_{80}$ In this section are presented the computational studies on the Wacker process by the analysis of the PES including solvent effects by combining explicit and implicit methods.

\section{Theoretical results supporting anti addition}

${ }_{85}$ The first theoretical calculation on the mechanism of the reaction was an extensive MO study. Eisenstein and Hoffmann studied this process using the Extended Hückel method in the context of examining nucleophilic additions to coordinated olefins to several transition metals. They showed that $\eta^{2}$ to $\eta^{1}$ 90 ethene slipping was the driving force toward the external nucleophilic attack. ${ }^{31}$ They also suggested that cis direct nucleophilic attack on the carbon is not favored; cis addition likely originates from addition to the metal followed by insertion of the olefin into the metal-nucleophile bond. Bäckvall and 95 coworkers $^{32}$ analyzed the stereochemistry of nucleophilic addition of several nucleophiles $\left(\mathrm{Nu}=\mathrm{CH}_{3}{ }^{-}, \mathrm{OH}^{-}{ }^{-} \mathrm{H}^{-}, \mathrm{F}^{-}\right)$to $\left[\mathrm{PdNu}_{2}\left(\mathrm{C}_{2} \mathrm{H}_{4}\right)\left(\mathrm{H}_{2} \mathrm{O}\right)\right]$ by thoughtfully relating ab initio $\mathrm{MO}$ calculations with the orbital interaction concept. The results indicated that a frontier-controlled cis migration to the olefin 100 would be possible only for coordinated $\mathrm{CH}_{3}{ }^{-}$and $\mathrm{H}^{-}$, whereas $\mathrm{OH}^{-}$and $\mathrm{F}^{-}$would be too unreactive in such a process. A related study performed by Fujimoto and Yamasaki on the hydroxypalladation of ethene also supported trans addition for hydroxide. ${ }^{33}$

105 After these initial studies Siegbahn was the first to tackle the Wacker process using DFT calculations including solvent effects by means of explicit and implicit models. ${ }^{34}$ The initially selected model reaction was the nucleophilic substitution between $\mathrm{PdCl}_{2}\left(\mathrm{C}_{2} \mathrm{H}_{4}\right)$ and $\mathrm{OH}^{-}$. The results were quite surprising. The 110 outer sphere attack of $\mathrm{OH}^{-}$on the olefin giving rise to $\left[\mathrm{Pd}(\mathrm{Cl})_{2}\left(\mathrm{C}_{2} \mathrm{H}_{4} \mathrm{OH}\right)\right]^{-}$, was highly exothermic, $454.4 \mathrm{~kJ} / \mathrm{mol}$. However, the attack on the $\mathrm{Pd}(\mathrm{II})$ center to form $\left[\mathrm{PdCl}_{2}(\mathrm{OH})\left(\mathrm{C}_{2} \mathrm{H}_{4}\right)\right]^{-}$was predicted to be even more favorable by as much as $209 \mathrm{~kJ} / \mathrm{mol}$ compared to the previous reaction. These 115 results were quite unrealistic and were attributed to artifacts due 
to the lack of explicit account of the water solvent.

One of the main deficiencies of the above computational strategy was that solvation effects were ignored. ${ }^{34}$ Inclusion of solvent effects by continuum methods had little effect on the 5 trends. Nucleophilic attack on the Pd(II) center was still largely favored over the attack on the alkene. The calculated reaction energies improved significantly by adding $\mathrm{H}^{+}$in the model, but this resulted in $\mathrm{Pd}-\mathrm{H}$ bond formation (thus generating a $\mathrm{Pd}(\mathrm{IV}$ ) intermediate) which is a clear artifact of the model. These 10 artifacts were avoided by employing $\mathrm{H}_{3} \mathrm{O}^{+}$instead of $\mathrm{H}^{+}$. This model had a significant impact on reaction energies favoring the $\mathrm{OH}^{-}$attack on the metal by $48.5 \mathrm{~kJ} / \mathrm{mol}$. These calculations showed that the presence of $\mathrm{H}_{3} \mathrm{O}^{+}$significantly improve such model.<smiles></smiles>

model complex including 3 waters

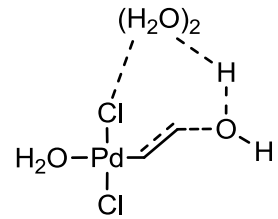

TS anti addition

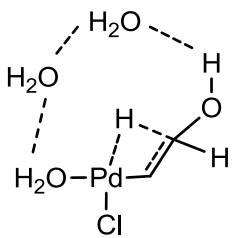

TS $\beta$-H elimination
Scheme 6 Model reaction steps calculated by Siegbahn including water molecules explicitly. ${ }^{34}$

The presence of additional water molecules in the model (two, three and four) was shown to be a more reliable model. ${ }^{35}$ ${ }_{20}$ By modeling the crucial nucleophilic addition and the $\beta$ elimination steps using these models, Siegbahn concluded that at least three water molecules are needed for reliable results (Scheme 3). The nucleophilic addition along with the formation of the $\mathrm{H}_{5} \mathrm{O}_{2}^{+}$takes place in a concerted way featuring a low 25 barrier $(23.8 \mathrm{~kJ} / \mathrm{mol})$. In fact, the intermediate is described as an $\mathrm{H}_{5} \mathrm{O}_{2}{ }^{+}-\mathrm{Cl}^{-}$ion pair (with such chloride ligand coordinated to the Pd(II) complex); see Scheme 6. The nucleophilic addition through a syn attack was also investigated and was found to be unfavorable compared to the anti addition. For the first time, ab

30 initio calculations along with a combination of explicit and implicit methods for representing the solvent concluded that the anti addition is the energetically preferred route. Moreover, the low energy barriers obtained compared to other steps indicated that the nucleophilic addition is not the rate determining step.

35 Most importantly, Siegbahn showed that due to charge separation occurring during the nucleophilic addition of water to ethene, inclusion of long range polarization effects of the solvent is required to obtain correct energetics.

Interestingly, the $\beta$-elimination process occurring subsequent 40 to the hydroxypalladation step was found to be the ratedetermining step by the same author. ${ }^{35}$ Again, the inclusion of explicit and implicit water models was inevitable to obtain reasonable free energy barriers $(55.6 \mathrm{~kJ} / \mathrm{mol})$. However this couldn't explain the KIE experiments where no primary isotope 45 effects were observed.

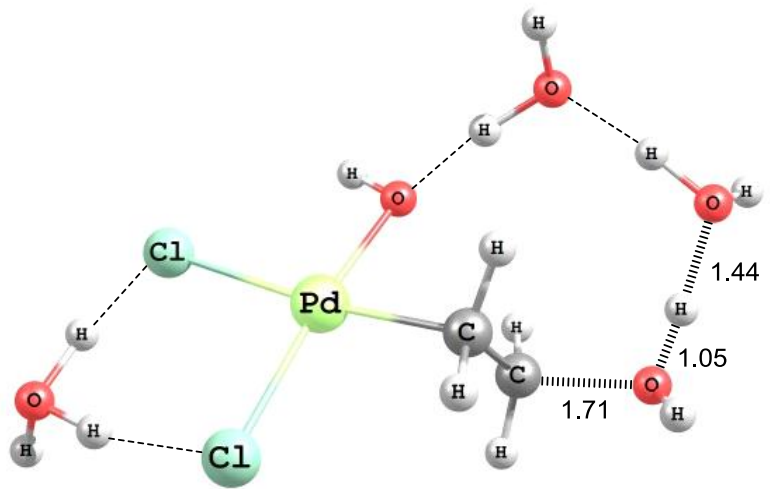

Fig. 1 Geometry of the anti transition state calculated by combining explicit (3-water chain) and implicit (PCM) model. ${ }^{36}$

In a later theoretical study Eshtiagh-Hosseini and coworkers 50 analyzed the reaction mechanism using a relatively similar theoretical model: ${ }^{36}$ cis- $\left[\mathrm{PdCl}_{2}(\mathrm{OH})\left(\mathrm{C}_{2} \mathrm{H}_{4}\right)\right]^{-}$along with $\mathrm{H}_{3} \mathrm{O}^{+}$ species forming an ion-pair with the chloride ligands. The model was completed with a chain of three water molecules (Fig. 1). Rate laws derived by assuming hydroxypalladation as the rate${ }_{55}$ determine step couldn't reproduce the experimentally observed rate-law at low $\left[\mathrm{Cl}^{-}\right]$and high $\left[\mathrm{Cl}^{-}\right]$. Thus, this study has been criticised by others. ${ }^{37}$ In the follow-up study, the same authors have looked at steps following the hydroxypalladation till the full oxidation of alkene. ${ }^{38}$ In this study, they assumed cis- $\mathrm{Cl}{ }^{-}$bond ${ }_{60}$ dissociation occurring subsequent to the hydroxypalladation, although the barrier for this process was not computed. This $\mathrm{Cl}$ dissociation step was required to have the cis position vacant for $\beta$-hydrogen to shift there. The authors have not thought along the lines of ligand isomerization, namely a possible cis-to-trans $\mathrm{Cl}$ 65 movement. From the overall results ${ }^{36,38} \beta$-hydride elimination is identified as the rate determining step. An important result of their work is that the conversion of $\left[\mathrm{Pd}(\mathrm{II}) \mathrm{Cl}\left(\mathrm{CH}_{3}-\mathrm{CH}-\mathrm{OH}\right)\right]$ to $\left[\mathrm{Pd}(0) \mathrm{Cl}\left(\mathrm{CH}_{3}-\mathrm{CHO}\right)\right]^{-}$involves a direct reductive elimination, in accordance with the results of Goddard and co-workers (see 70 below), ${ }^{39}$ but assisted by solvent water molecules instead of a $\beta$ hydride elimination or direct elimination to $\mathrm{Cl}^{-}$ligand.

The reaction mechanism for the Wacker process catalyzed by palladium acetate in acetic acid was studied by Kratgen et al. ${ }^{40}$ In their model it is assumed that the catalyst is formed by a $\mathrm{Pd}(\mathrm{II})$ 75 dimer with two acetates bridging the metals and other acetates or water molecules fulfilling the coordination sphere, along with a continuum method for the solvent. The authors evaluated both, the syn and anti mechanisms, concluding that the latter is favored.

\section{Theoretical results supporting syn addition}

The first theoretical study supporting the syn nucleophilic addition was performed by Nelson et al. by means of MNDO semiempirical calculations. ${ }^{11}$ As already commented in the previous section, correlation between reaction rates with 85 ionization potentials and HOMO and LUMO energies on a series of alkenes, was interpreted by the authors as a support for the syn addition.

Later, Oxgaard, Goddard and co-workers have also studied this process on the basis of theoretical calculations trying to 90 rationalize the dependence of the addition mode on reaction conditions. ${ }^{41}$ They stressed the importance of solvent effects, 
especially the necessity of accurate estimation of solvation entropy. Although these authors used an implicit water model with only one solvent water molecule, they added empirical corrections to the Gibbs energy of solvation of ions to tally the 5 experimental estimates, which in turn influenced the prediction of the mechanism. Hence, a correction was included in the calculations to obtain reasonable energy barriers for those steps where a proton is released to the medium (forming a hydronium species), this is for both the anti and syn transition states. ${ }^{41}$ This 10 shows that the poor description of the solvation energy of $\mathrm{H}_{3} \mathrm{O}^{+}$ within the implicit solvation model requires additional, a posteriori corrections in order to obtain reliable values for proton releasing processes.

The barrier of the anti nucleophilic addition to cis$15\left[\mathrm{PdCl}_{2}\left(\mathrm{C}_{2} \mathrm{H}_{4}\right)\left(\mathrm{H}_{2} \mathrm{O}\right)\right]$ was found to be quite low $\left(\Delta \mathrm{H}^{\neq}=36.4\right.$ $\mathrm{kJ} / \mathrm{mol}$ ) in qualitative agreement with previous calculations in the literature. Nevertheless, by incorporating entropic effects by means of a more complex treatment (including empirical corrections) a significant increase of the Gibbs energy barrier was 20 observed up to $78.2 \mathrm{~kJ} / \mathrm{mol}^{41}$ (still below of the experimental energy barrier of $101.2 \mathrm{~kJ} / \mathrm{mol}$ ). According to these numbers, in principle, such barrier corresponds to a feasible step within the reaction. On the other hand, the syn addition was also investigated. Prior to this process the formation of the cis$25\left[\mathrm{PdCl}_{2}(\mathrm{OH})\left(\mathrm{C}_{2} \mathrm{H}_{4}\right)\right]^{-}$intermediate by a proton release from the aqua ligand takes place. The energy barrier for the syn hydroxypalladation step was found to be $139.6 \mathrm{~kJ} / \mathrm{mol}$, also too high to be a feasible reaction step. Clearly, these theoretical studies seriously questioned the feasibility of the originally 30 proposed syn addition in the Wacker process.
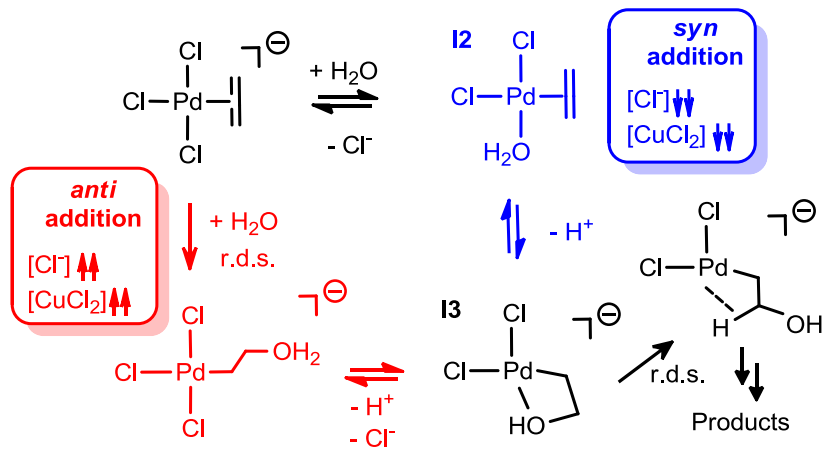

Scheme 7. Reaction mechanisms proposed by Goddard et al. ${ }^{41}$

These authors found an alternative, energetically feasible pathway for the syn addition (Scheme 7). ${ }^{41}$ The initial steps in the 35 reaction are the same, substitution of two $\mathrm{Cl}^{-}$by $\mathrm{C}_{2} \mathrm{H}_{4}$ and $\mathrm{H}_{2} \mathrm{O}$, respectively to form cis- $\left[\mathrm{PdCl}_{2}\left(\mathrm{C}_{2} \mathrm{H}_{4}\right)\left(\mathrm{H}_{2} \mathrm{O}\right)\right]$; the associated energy barrier is $97.4 \mathrm{~kJ} / \mathrm{mol}$. At this point the authors envisaged a process where a water molecule simultaneously deprotonates the coordinating water in $\mathbf{I} 2$ to form palladacycle $\mathbf{I 3}$ (see Scheme 40 7). This process has an energy barrier of $75.2 \mathrm{~kJ} / \mathrm{mol}$. Subsequently, a rotation around the $\mathrm{C}-\mathrm{C}$ bond converts the palladacycle in an intermediate with an agostic interaction. The barrier for this conversion was found to be $97.0 \mathrm{~kJ} / \mathrm{mol}$, and was considered to be the potential rate determining step. This 45 assumption was necessary to bring into accord the predicted route with the experimental rate law corresponding to low $\left[\mathrm{Cl}^{-}\right]$.

In their work the authors tried to rationalize all their results according to the reaction conditions and their respective rate laws. Thus, the mechanism described so far is for low $\left[\mathrm{Cl}^{-}\right]$and ${ }_{50}\left[\mathrm{CuCl}_{2}\right]$. For the case of high concentration of these species they considered an alternative mechanism with an anti addition of $\mathrm{H}_{2} \mathrm{O}$ to $\left[\mathrm{PdCl}_{3}\left(\mathrm{C}_{2} \mathrm{H}_{4}\right)\right]^{-}$(Scheme 7). The rate determining step is in this case the nucleophilic addition of a solvent water to the complex to generate $\left[\mathrm{PdCl}_{3}\left(\mathrm{CH}_{2} \mathrm{CH}_{2} \mathrm{OH}\right)\right]^{-}$with an energy 55 barrier of $94.9 \mathrm{~kJ} / \mathrm{mol}$. In a subsequent step there is a proton release along with the formation of palladacycle I3, the intersection point between the syn and anti mechanisms. Overall, these authors propose two main mechanisms for the Wacker process depending on the reaction conditions, as is expected from ${ }_{60}$ the differences in the rate laws associated with different chlorine concentrations.

To complete the generation of acetaldehyde several $\mathrm{H}$ transfers are subsequently needed. Goddard and co-workers were the first to point out that $\beta$-hydride elimination from - $\mathrm{OH}$ groups ${ }_{65}$ is not accessible (energy barrier of $151.3 \mathrm{~kJ} / \mathrm{mol}$ ). ${ }^{3}$ Instead, they propose an alternative through a chloride-mediated reductive elimination where $\mathrm{HCl}$ and $\mathrm{Pd}(0)$ form directly. Later, EshtiaghHosseini et al. published a quite similar mechanism with analogous conclusions for the H-transfer processes (Fig. 2), 70 where the proton was abstracted by water molecules hydrogenbonded to chloride. ${ }^{38}$
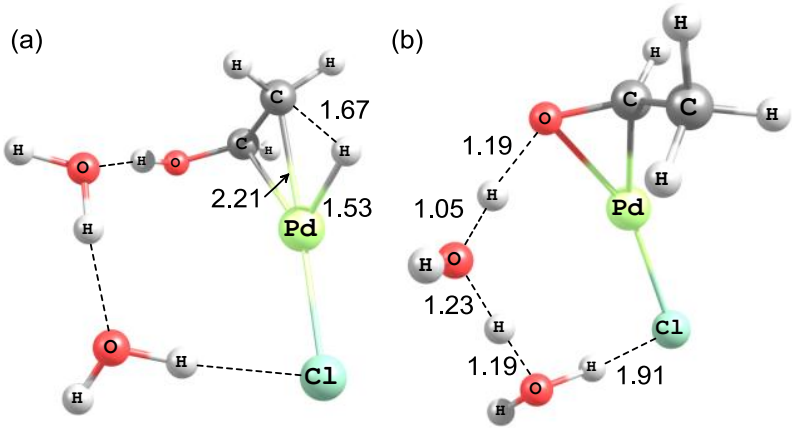

Fig. 2 Calculated TS for $\mathrm{H}$-transfer processes: (a) alkene insertion into the $\mathrm{Pd}-\mathrm{H}$ bond, (b) $\beta-\mathrm{H}$ elimination from $-\mathrm{OH}$ functional group. Atomic 75 distances are in $\AA$.

\section{General overview on dynamic calculations}

From all theoretical calculations reviewed so far, it is clear that increasing the number of water molecules to "microsolvate" the complex will complicate the energy estimates due to the 80 existence of several structural manifolds with nearly identical potential energies. Indeed, when the reaction involves many atoms and especially solvent participates actively in the mechanism as in the Wacker process, the underlying PES becomes very complicated with a plethora of minima and saddle 85 points within a few $\mathrm{kJ} / \mathrm{mol}$ heights. The majority of these stationary points are dynamically irrelevant at finite temperature and their exploration and evaluation is both time-consuming and unnecessary. Moreover, boundary effects due to finite size of solvation can also result in erroneous predictions. Therefore, it is 90 most important to explicitly account for the entropic contribution and finite temperature effects due to the solvent used. The strategy in such cases is to turn to the free energy surface (FES) of the reaction as a function of a few reaction coordinates. The 
FES can be calculated from the probability distribution $P(\mathbf{s})$ of the system: ${ }^{42}$

$$
F(\mathbf{s})=-R T \ln [P(\mathbf{s})]
$$

where $\mathbf{s}$ is the vector of a few (usually 1-3) coordinates. $F(\mathbf{s})$ in ${ }_{5}$ Eq. 9 is defined up to a constant, which however is unimportant as we are interested in relative free energies. The probability distribution is obtained from canonical sampling, keeping $N, V$ and $T$ (number of particles, volume and temperature) fixed. Kinetics and thermodynamics observables can be calculated from 10 the properties of the FES: the minima are metastable reactant and product states whereas the saddle points between these minima are the transition states associated with the kinetic bottleneck of the reaction. Exploration of the FES can be done by Monte-Carlo (MC) or Molecular Dynamics (MD) simulations. If the system is 15 ergodic in the time-scale of the simulations, both methods yield the same FES. An important feature of the MD simulations as compared to MC is that they follow the actual dynamical evolution of the system which is essential in elucidating reaction mechanisms. In fact, in the last decades ab initio molecular 20 dynamics (AIMD) has become a standard tool for exploring reactive transformations. ${ }^{42}$ In these simulations the underlying quantum chemical equations are solved by using DFT methods, as they provide an attractive compromise between accuracy and computational cost. With the aid of this approach, simulations 25 can be carried out to model finite solvent molecules within a periodic box mimicking the bulk solvent environment at a finite temperature (and density). Using a plane-wave basis set is more appropriate for such calculations as by their construction the wavefunction of the system within this basis is periodic. Plane30 wave DFT based molecular dynamics simulations are therefore well suited to study chemical reactions like the Wacker process that occur in solutions. These methods are however computationally expensive and their application is plagued by a serious conceptual problem: A finite, standard MD calculation

35 employing DFT can access at most tens of picoseconds timescale due to its high CPU demand. In addition in these simulations only low energy (metastable) regions can be sampled sufficiently, whereas high-energy regions are sampled very rarely. When a free energy barrier, significantly larger than a few ${ }_{40}$ RT-s, separates the reactant and product configurations, the time between transitions from one metastable state to another can be orders of magnitudes longer than the molecular vibrational period. Therefore observation of even a single transition in conventional MD simulation is highly unlikely and direct 45 sampling of the transition state area is not feasible. This is the well-known time-scale problem, i.e. the presence of a characteristic gap between the accessible time-scales and those of interest. $^{43}$

A large number of methods have been developed to make 50 these rare events occur more frequently (see Ref. 43 for a representative list). The most efficient methods for mechanistic studies enhance the samplings and explore the FES as a function of a few predefined reaction coordinates. An important methodological issue is that the a priori choice of a set of 55 appropriate reaction coordinates is usually far from obvious and is largely governed by chemical or physical intuition. In general, a suitable set of reaction coordinates (or in more general terms, collective variables, CV-s) should be able to distinguish between reactant, product and transition states, and they have to cover all ${ }_{60}$ the slow degrees of freedom coupled with the reaction ${ }^{43}$. When these requirements are not fulfilled, the calculated FES will be incorrect and cannot be used to interpret reaction mechanisms. Since we wish to keep the number of CV-s low, we need coordinates as general as possible. Typical examples are bond ${ }_{65}$ distance, angles, coordination numbers, path variables, etc. ${ }^{43}$

A very convenient method for exploring FES is metadynamics. ${ }^{43}$ Metadynamics (MTD) employs a bias potential acting on predefined CV-s $(\boldsymbol{s})$, and it is history dependent:

$$
V(\boldsymbol{s}, t)=\sum_{t^{\prime} \leq t} w \exp \left(-\frac{\left(s-s\left(t^{\prime}\right)\right)^{2}}{2 \sigma^{2}}\right)
$$

70 i.e. it is a sum of Gaussians deposited along the trajectory of the system at regular time intervals. The parameters $w$ (Gaussian height), $\sigma$ (Gaussian width) and the deposition frequency determine the efficiency and accuracy of MTD. The role of the history dependent potential is to encourage the system to visit 75 unexplored regions on the FES. The deposited Gaussians fill up the first metastable basin (reactant state) and force the system to escape to the next metastable region (product state) through the lowest energy barrier. A very important feature of the method is that after a sufficient time the bias potential provides an unbiased 80 estimate of the underlying free energy surface: ${ }^{43}$

$$
-\lim _{t \rightarrow \infty} V(\boldsymbol{s}, t) \simeq F(\boldsymbol{s})
$$

From the available free energy techniques that can be used to boost the activated ligand exchanges, all the studies of the Wacker reactions have unequivocally selected the metadynamics 85 method. The reason is that MTD offers several advantages. It can be used for finding the lowest saddle point driving the system from one state to another ("escaping free energy minima"). It can efficiently explore reactions consisting of more than one elementary step and their sequence can be determined, i.e. the 90 reaction mechanism in the space of the preselected coordinates can be reconstructed or proved. It provides large flexibility in selecting, combining and treating $\mathrm{CV}$-s, it can reveal new, unexpected reaction paths, the results can be systematically improved and finally the present implementations are very easy 95 to use and often chemically insightful results can be obtained even from a single run.

Experience shows that MTD can be very efficient in exploring multidimensional coordinate space and can locate the most probable reaction path at the expense of low accuracy. Varying 100 systematically the parameters of the history dependent potential allows to converge the results to the required accuracy. MTD, similarly to other free energy methods, is a statistical method; hence the error of the calculated free energy values can be estimated and systematically improved. In typical metadynamics 105 simulations employed in catalytic modelling the activation energies can have errors of up to $5-6 \mathrm{~kJ} / \mathrm{mol}$ while the reaction free energies can have statistical errors up to $12 \mathrm{~kJ} / \mathrm{mol}$ if they are calculated as the difference between two activation free energy values, due to error propagation. These values transform to an 110 order of magnitude ( 11 -fold) difference in the calculated TST rates, and two orders of magnitude (123-fold) difference in the calculated equilibrium constants at ambient temperature. An 
alternative is that the coarse FES can be further refined by other free-energy methods, such as umbrella sampling along the path, combining the advantages of the two methods. ${ }^{43}$

\section{Free energy simulations on the Wacker reaction steps}

${ }_{5}$ One of the most important issues in exploring the mechanism of the Wacker reaction is the nature of the catalytically active $\mathrm{Pd}(\mathrm{II})$ species. Experiments firmly established that under typical Wacker conditions the $\mathrm{PdCl}_{2}$ reactant spontaneously transforms to the catalyst precursor $\left[\mathrm{PdCl}_{4}\right]^{2-}(\mathbf{1})$. The chemical reactivity of ${ }_{10} \mathbf{1}$ is crucial for the mechanism. On one hand, the rate laws for both the high and low $\left[\mathrm{Cl}^{-}\right]$cases indicate ligand exchanges in the $\mathrm{Pd}(\mathrm{II})$ ligand sphere preceding the rate determining step, ie. the catalytic route strongly depends on the stoichiometry of the catalytically active Pd(II) species. On the other hand, the actual 15 configuration of the catalytically active $\mathrm{Pd}(\mathrm{II})$ compound is strongly dependent on these substitutions. Indeed, there is a very strong organizing force which severely constrains the actual arrangement of the ligands around the $\mathrm{Pd}(\mathrm{II})$ ion, namely the trans-effect. The trans-effect is the ability of a ligand bonded to a 20 transition-metal ligand sphere to destabilize the bond in trans position (trans influence) or to increase the rate of the substitution in trans position (kinetic trans effect). The $\mathrm{H}_{2} \mathrm{O}, \mathrm{Cl}^{-}$ and $\mathrm{C}_{2} \mathrm{H}_{4}$ ligands have characteristically different trans-effects. $\mathrm{C}_{2} \mathrm{H}_{4}$ has a very high trans-effect, whereas $\mathrm{Cl}^{-}$and $\mathrm{H}_{2} \mathrm{O}$ have 25 medium and low effects, respectively. It is important to note that the trans-effect is a local phenomenon operating in the first ligand-sphere of the transition-metal center. This implies that it does not depend on the ligand concentration. An important consequence is that for the Wacker reaction the trans-effect is 30 operative at both high and low $\left[\mathrm{Cl}^{-}\right]$.

Ligand exchanges in solution represent typical processes which require advanced modelling strategies. This is because there are various effects which cannot be described with simple gas-phase models: significant charge displacements, solvent 35 reorganizations, large configurational freedom and other entropic effects. The adequate strategy in such cases is to employ AIMD.

An important element in constructing the overall kinetics and the mechanism is verifying whether the ligand exchanges are equilibrium processes. Kinetics experiments suggested that the 40 steps preceding the hydroxypalladation do indeed represent equilibria and the simulations ${ }^{44,45,46}$ have strongly supported these assumptions in all fronts.

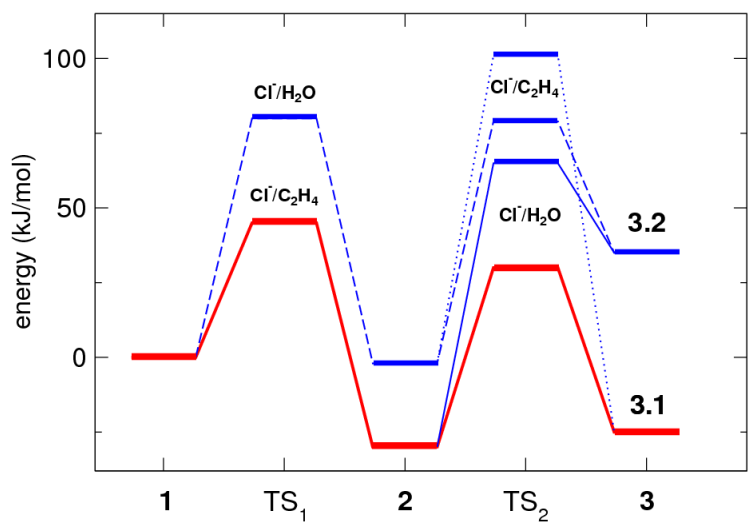

Fig. 3 Free energy profiles for the various ligand exchanges leading to the 45 formation of the trans- and cis- $\left[\mathrm{PdCl}_{2}\left(\mathrm{C}_{2} \mathrm{H}_{4}\right)\left(\mathrm{H}_{2} \mathrm{O}\right)\right]$ (3.1 and 3.2) complexes. Red: the optimal $\mathbf{1} \rightarrow \mathbf{2} \rightarrow \mathbf{3 . 1}$ route; blue solid line: $\mathbf{1} \rightarrow \mathbf{2} \rightarrow$ 3.2 path; blue dashed line: $\mathbf{1} \rightarrow \mathbf{2}^{\prime} \rightarrow \mathbf{3 . 2}$ route; blue dotted line: $\mathbf{1} \rightarrow \mathbf{2}^{\prime}$ $\rightarrow 3.1$ path.

50 The ligand exchanges on the catalyst precursor 1 were initially studied by Nair and coworkers, ${ }^{44,45}$ and Kovács et al. ${ }^{46}$ In all these studies, it is shown that ligand exchanges take place in a stepwise fashion. The experimental rate law indicates two ligand substitutions at low $\left[\mathrm{Cl}^{-}\right]$: a $\mathrm{C}_{2} \mathrm{H}_{4}$ and a water ligand replace two ${ }_{5} \mathrm{Cl}^{-}$anions. There are two possible sequences for these processes: either first the $\mathrm{Cl}^{-} / \mathrm{H}_{2} \mathrm{O}$ exchange takes place followed by the $\mathrm{Cl}^{-}$ $/ \mathrm{C}_{2} \mathrm{H}_{4}$ substitutions, or the other way around. A complication arises from the competition between the trans-effects of the first entering substituent and the remaining $\mathrm{Cl}^{-}$ligands and this in fact 60 eventually determines the configuration of the $\left[\mathrm{PdCl}_{2}\left(\mathrm{C}_{2} \mathrm{H}_{4}\right)\left(\mathrm{H}_{2} \mathrm{O}\right)\right]$ species formed in the second step. Fig. 3 shows the free energy profiles for all the four possible paths leading to the formation of the trans- and cis- $\left[\mathrm{PdCl}_{2}\left(\mathrm{C}_{2} \mathrm{H}_{4}\right)\left(\mathrm{H}_{2} \mathrm{O}\right)\right]$ (3.1 and 3.2) complexes. While some discrepancies appear 65 between these papers among some of the calculated activation free energies owing to the differences in the actual models and in the technical details, the conclusions regarding the chemical reactivity of $\mathbf{1}$ are consistent with the picture shown in Fig. 3. The essential result of the ligand-exchange simulations is that the 70 most likely pathway is the $\mathbf{1} \rightarrow\left[\mathrm{PdCl}_{3}\left(\mathrm{C}_{2} \mathrm{H}_{4}\right)\right]^{-}(\mathbf{2}) \rightarrow \mathbf{3 . 1}$ route, from both kinetic and thermodynamic points of view, whereas the other routes can be safely excluded. This crucial result points to an interesting chemistry occurring in the Pd(II)-ligand sphere and its rationalization seems very important for a deeper 75 understanding of the properties of catalytically active $\operatorname{Pd}(\mathrm{II})$ complexes.

The first ligand exchange is both kinetically and thermodynamically more favorable when a $\mathrm{C}_{2} \mathrm{H}_{4}$ substitutes a chlorine anion as opposed to the $\mathrm{H}_{2} \mathrm{O} / \mathrm{Cl}^{-}$exchange (Scheme 8). ${ }_{80}$ We note that the simulations nicely reproduced the experimental trends in the stability of the monosubstituted complexes. ${ }^{7,47}$ The higher stability of $\mathbf{2}$ can be explained by the favorable $\pi$-binding of the ethene ligand. The barriers of the first ligand exchange show the same trend following from the Hammond-postulate. In 85 the second step, another chloride ligand is replaced by either a water or an ethene ligand. At this point however four possible channels can be considered. As Fig. 3 shows, the route $\mathbf{2} \rightarrow \mathbf{3 . 1}$ features the smallest activation energy and within the error margin of the simulations it is thermodynamically an almost 90 neutral equilibrium, slightly shifted toward the $\mathbf{2}$ state. In contrast, the formation of the cis complex is thermodynamically very unfavorable and preceded by quite substantial free energy barriers from both the 2 and the $\left[\mathrm{PdCl}_{3}\left(\mathrm{H}_{2} \mathrm{O}\right)\right]^{-}\left(\mathbf{2}^{\prime}\right)$ directions. Curiously, the largest activation free energy can be found along 95 the $\mathbf{2}^{\prime} \rightarrow \mathbf{3 . 2}$ path $(\sim 100 \mathrm{~kJ} / \mathrm{mol})$. Comparison of the profiles reveals the role of the strong trans-effect of $\mathrm{C}_{2} \mathrm{H}_{4}$ in the ligand sphere: the substitution leading to trans complex features a much smaller activation barrier than the one toward the cis complex. On the other hand, as chloride has a stronger trans-effect than 100 water, the barrier is higher when the $\mathrm{C}_{2} \mathrm{H}_{4} / \mathrm{Cl}$ exchange occurs in trans with respect to the water; ie. the barrier is lower when the substitution is trans to $\mathrm{Cl}^{-}$. 

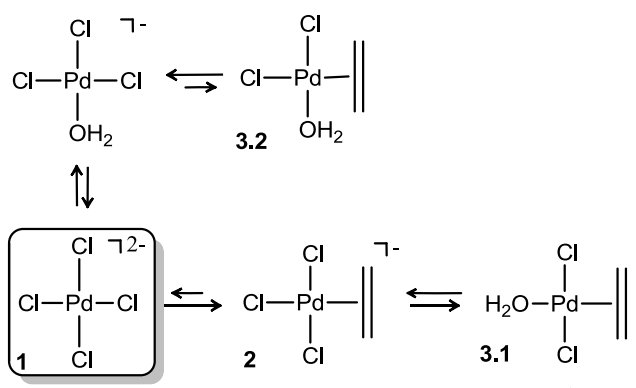

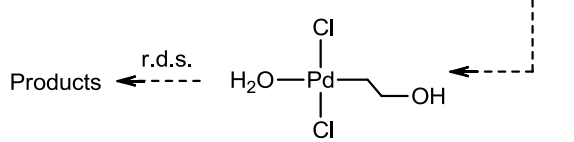

Scheme 8 Ligand exchanges on the catalyst leading to the the cisand trans- $\left[\mathrm{PdCl}_{2}\left(\mathrm{C}_{2} \mathrm{H}_{4}\right)\left(\mathrm{H}_{2} \mathrm{O}\right)\right]$ complexes.

An alternative route for the formation of the cis isomer goes 5 via trans-to-cis isomerization process, as suggested by Jira and others. ${ }^{16}$ In this route the trans-complex undergoes another $\mathrm{Cl}^{-}$ $/ \mathrm{H}_{2} \mathrm{O}$ exchange in one of the cis positions to ethene, then the $\mathrm{H}_{2} \mathrm{O}$ $/ \mathrm{Cl}^{-}$exchange takes place at the trans position giving rise to the cis isomer. Nevertheless, the activation free energies have been 10 calculated to be 110 and $40 \mathrm{~kJ} / \mathrm{mol}$ in the forward direction. ${ }^{44,45}$ In addition, the high energy difference between the cis and trans complex has to be surmounted. Clearly, this route can also be excluded from the mechanism according to these calculations.

Regarding the stabilities of the cis- and trans$15\left[\mathrm{PdCl}_{2}\left(\mathrm{C}_{2} \mathrm{H}_{4}\right)\left(\mathrm{H}_{2} \mathrm{O}\right)\right]$ it can be seen that formation of the transisomer is exergonic and much more favourable then the formation of the cis complex. In fact, the simulations predict a 60 $\mathrm{kJ} / \mathrm{mol}$ free energy difference between the cis and trans configurations. This is equivalent to an equilibrium constant of $203 \cdot 10^{10}$ for the cis $\leftrightarrow$ trans transformation at room temperature. Hence under equilibrium conditions the concentration of the $\mathrm{cis}$ isomer is negligible.

The results of the ligand exchange simulations have profound consequences for the reaction mechanism. The inner-sphere, syn 25 hydroxypalladation can play a role if it has a very low activation barrier to compensate the extremely low concentration of the $\mathbf{3 . 2}$ species. On the other hand the outer-sphere, intermolecular (anti) hydroxypalladation can occur on both the cis and trans configurations and the subsequent free energy simulations have 30 indeed showed that these processes are feasible.

Hydroxypalladation reaction was calculated for both 3.1 and 3.2 isomers. $^{44,45,48}$ The former will only proceed through an outersphere mechanism, as the cis-position is occupied by chlorine. The free energy barrier estimated by metadynamics is 35 about $75 \mathrm{~kJ} / \mathrm{mol}$ for this reaction. These simulations also provided crucial details of the reaction at molecular level. Olefin undergoes a slipping motion from the equilibrium $\eta^{2}$ coordination to (nearly) $\eta^{1}$ coordination before the water addition. More than thirty years back, through molecular orbital analysis of transition metal 40 complexed olefines, Eisenstein and Hoffmann ${ }^{31}$ predicted that such a slipping motion of olefin is playing a vital role in activating olefins. Analysis of the reactive trajectory also showed that the deprotonation of the attacking water molecule and $\mathrm{C}-\mathrm{O}$ bond formation occur simultaneously. The proton of the attacking 45 water was seen moving out to the bulk subsequently. There seems to be a consensus on these results obtained by all the three technically different AIMD simulations. ${ }^{44,45,48}$ It is worth pointing out at this stage that such an unbiased sampling of reactive trajectories is only possible by combining ab initio molecular 50 dynamics with metadynamics.

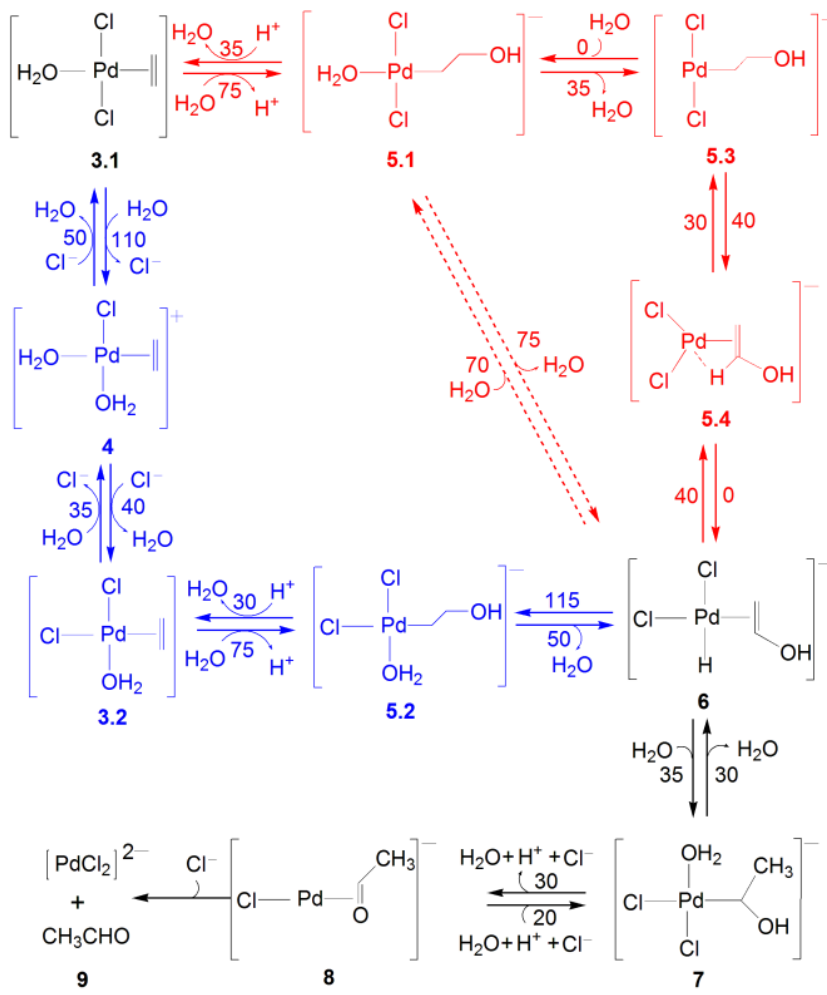

Fig. 4 Reaction mechanism of the oxidation of ethene at low [Cl-]. Paths $\mathrm{A}$ and $\mathrm{B}$ are highlighted in red and blue colors, respectively; see Ref. 45 for details

55 Despite the formation of the $\mathbf{3 . 2}$ was ruled out by Kovács et al., ${ }^{46}$ Nair and co-workers revaluated such process obtaining similar results. ${ }^{45}$ Hydroxypalladation of $\mathbf{3 . 2}$ was also carried out in such a way that both inner-sphere and outer-sphere mechanisms are sampled in the metadynamics simulation. ${ }^{48}$ It 60 was found that the reaction proceeded through an outer sphere mechanism, exactly in the same manner as $\mathbf{3 . 1} \rightarrow \mathbf{5 . 1}$. When a metadynamics simulation was carried out by restricting the reaction to proceed only through the inner sphere mechanism, the free energy barrier for the reaction was found to be $\sim 200 \mathrm{~kJ} \mathrm{~mol}^{-}$ $65{ }^{148}$ The difference in the free energy barriers between the two routes is much beyond the error in the free energy estimates associated with the methods employed. Further, formation of $\mathbf{3 . 2}$ has to occur through a cis $\mathrm{Cl}^{-}$ligand-exchange with bulk water, $3.1 \rightarrow 4$, which has to overcome a barrier of about $110 \mathrm{~kJ} \mathrm{~mol}^{-1}$ 70 and thus is slower than the hydroxypalladation of 3.1. Thus, AIMD simulations clearly rule out the occurrence of the inner sphere mechanism in the Wacker oxidation of ethene. 


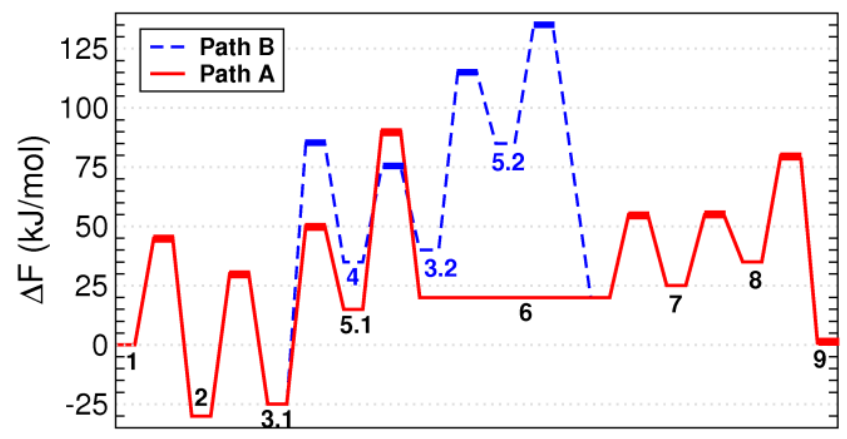

Fig. 5 Free energy profile for the Wacker oxidation reaction of ethene as shown in Ref. 45.

Due to the attack of a water molecule from the bulk, outer 5 sphere hydroxypalladation is expected to be an equilibrium process, in contrast to a non-equilibrium step expected for the inner sphere mechanism.3 Reprotonation of the $\mathrm{OH}$ group of hydroxyethyl ligand by the proton from solvent should be a relatively fast process compared to any other steps following the 10 hydroxypalladation. Therefore, if the outer sphere mechanism is preferred, a rate-determining step should follow the hydroxypalladation such that the experimental rate-law can be explained. ${ }^{7}$ Bäckvall et al. ${ }^{15}$ proposed a ligand dissociation step after the hydroxypalladation as the rate-determining step. ${ }_{15}$ However, the isotope scrambling experiments with allyl-1,1-d2 alcohol indicates that hydroxypalladation is not an equilibrium step. ${ }^{18}$ As discussed before, earlier theoretical calculations6,36,38 proposed that $\beta$-hydrogen transfer following the hydroxypalladation step is the rate-determining, and the transition 20 state involves the dissociation of the $\mathrm{C}_{\beta}-\mathrm{H}_{\beta}$ bond, and formation of the $\mathrm{Pd}-\mathrm{H}_{\beta}$ bond. In such a case, a significant primary isotope effect is expected, but was not seen experimentally. ${ }^{10}$

To answer these perplexing questions, a full free energy ${ }_{25}$ profile of the Wacker process till $\mathrm{CH}_{3} \mathrm{CHO}$ formation was computed by extensive AIMD simulations. ${ }^{45}$ In order to obtain such a free energy profile, metadynamics simulations of both forward and backward reactions have to be carried out. Thus, 21 independent metadynamics simulations were needed to obtain the 30 free energy profiles along the entire reaction as shown in Fig. 4 and thus are computationally expensive. From the free energy profile, it is clear that the rate-determining step along Path $\mathrm{A}$ is $\mathbf{5 . 1} \rightarrow \mathbf{6}$, which is the $\beta$-hydrogen elimination. On analysis of the free energy surface (Fig. 5), it was observed that the step with the 35 highest free energy features an isomerization, and not the $\mathrm{H}_{\beta}$ transfer. The isomerization is in fact the movement of cis-Cl to the trans position after the trans water detachment. The $\mathrm{Pd}-\mathrm{H}_{\beta}$ transfer occurs immediately after the isomerization as a barrierless process. A few more metadynamics simulations of this 40 reaction step with different set of collective coordinates were also carried out to further confirm the aforementioned mechanism.

This is an unprecedented result, however, and it answers many of the open questions that we discussed above. According 45 to this mechanism, the rate-determining step does not involve $\mathrm{H}_{\beta}$ transfer and thus, the absence of a primary isotope effect can be explained. As can be seen from the snapshots in Fig 6, weak Hbond interactions are present between $\mathrm{Pd}$ and $\mathrm{H}_{\beta}$ in the transition state, and thus could be the reason for the observed secondary 50 isotope effect. ${ }^{10}$
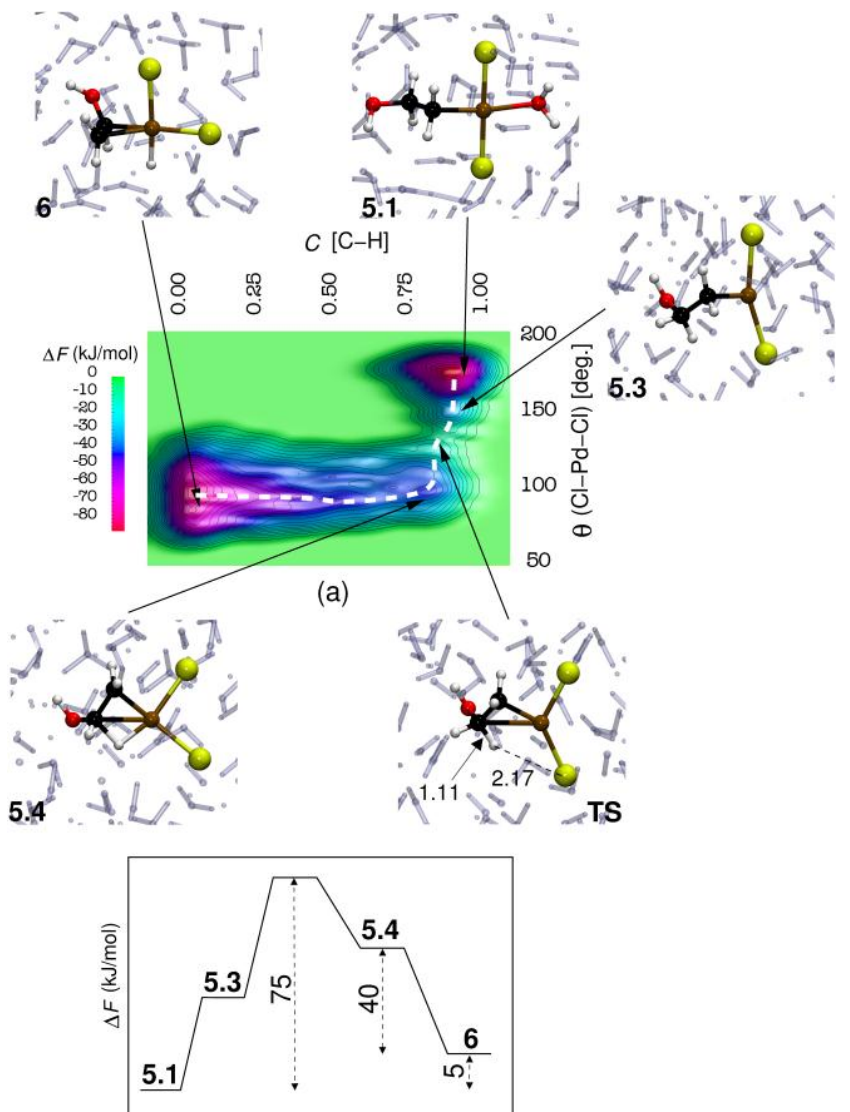

(b)

Fig.6 Free energy surface for $\mathbf{6} \rightarrow \mathbf{5 . 1}$; this surface also gives the nature of the $\mathbf{5 . 1} \rightarrow \mathbf{6}$ reaction. Some snapshots along the reactive trajectory is also 55 shown. TS indicates the likely transition state structure, and the bond distances are in $\AA$.

Another interesting mechanism observed is for the step $\mathbf{7 \rightarrow \mathbf { 8 }}$ which involves a deprotonation of the hydroxyethyl $\mathrm{OH}$ group, forming acetaldehyde. Different mechanisms are proposed in the 60 literature for this step: a) $\beta$-hydrogen elimination, ${ }^{49}$ (b) deprotonation to $\mathrm{Cl}^{-} ;{ }^{39}$ (c) or to a solvent water molecule. ${ }^{38} \mathrm{~A}$ metadynamics simulation was performed where all these three possibilities were accounted for. Simulations showed that deprotonation occurs to a solvent water molecule in agreement 65 with Ref. 38.

The overall free energy profile also indicates that, along Path A, the free energy barrier for the reaction is $90 \mathrm{~kJ} / \mathrm{mol}$, which is in excellent agreement with the experimental estimate of 94 ${ }_{70} \mathrm{~kJ} / \mathrm{mol}^{7}$ Path B can be ruled out as the effective barrier along this pathway is $135 \mathrm{~kJ} / \mathrm{mol}$. Having access to the overall free energy profile of the oxidation, one can derive the rate-expression for the process using the steady state approximation for the intermediate concentration. The rate-law for $\mathbf{1} \rightarrow \mathbf{9}$ was obtained as

75

$$
-\frac{d\left[\mathrm{C}_{2} \mathrm{H}_{4}\right]}{d t}=\frac{k_{\mathrm{eff}}\left[\mathrm{PdCl}_{4}^{2-}\right]\left[\mathrm{C}_{2} \mathrm{H}_{4}\right]}{\left[\mathrm{H}^{+}\right]\left[\mathrm{Cl}^{-}\right]^{2}}
$$


where $k_{\text {eff }}$ is the effective rate constant. ${ }^{45}$ This expression is exactly the same as observed experimentally. The origin of the first order proton inhibition can be traced back to the hydroxypalladation step, and the second order chloride inhibition 5 is due to the two $\mathrm{Cl}^{-}$ligand exchanges (i.e $\mathbf{1} \rightarrow \mathbf{2}$, and $\mathbf{2} \rightarrow \mathbf{3 . 1}$ ) occurring before the hydroxypalladation step. Nevetheless, we note here in passing that a direct comparison of the estimated effective free energy barrier with the experimentally determined free energy barrier has to be done with caution. Experimental free 10 energy barriers are estimated based on the turn-over-frequency, which can be influenced by the free energies of the steps subsequent to the oxidation of ethene (namely, oxidation of Pd and oxidation of $\mathrm{Cu}$ ). However, these steps have not been yet studied by AIMD simulations.

15

\section{Conclusions}

Taking the classic Wacker process as an example, we have shown the potential of AIMD in modeling homogenous catalysis. Since water acts as a solvent and a reagent in the Wacker process, it 20 poses several challenges to model these reactions through static quantum chemical approaches, even after including finite and continuum solvent models. By discussing the results of AIMD simulations, it is demonstrated that AIMD techniques allow one to go beyond the chemical intuitions, as appropriate for studying 25 reactions in solutions. These approaches also enable one to include finite temperature and explicit solvent effects very efficiently. By combining AIMD with enhanced sampling approaches like metadynamics, reaction mechanism and associated free energy changes along the minimum energy 30 pathway can be obtained.

AIMD simulations contributed to a plethora of mechanistic and kinetic information of the Wacker process. A proper understanding of the ligand exchanges, trans effect, 35 hydroxypalladation, and subsequent hydrogen transfer reactions were possible only because of the recent AIMD simulations. Unequivocally, AIMD is the most appropriate technique to study such complex homogenous catalysis.

${ }_{40}$ Several questions regarding the Wacker process are yet to be resolved: a) why was isotope scrambling of allyl-alcohol not observed in experiments if the rate-determining step follows the anti-mode hydroxypalladation? b) why were different rate-laws observed at different $\left[\mathrm{Cl}^{-}\right]$and what is the effect of $\left[\mathrm{Cl}^{-}\right]$on the 45 mechanism? c) Can dimerization of the catalytic precursor occur in solution, and does it influence the actual catalyst structure? We are planning to carry out our future work in these directions.

Although computer simulations modeling homogenous 50 catalysis with finite temperature and explicit water molecules are computationally expensive, with the modern developments in computer technology and low-latency networks it is now practically possible to carry out such calculations on High Performance Computers. In the coming years, we can thus 55 anticipate plenty of applications of AIMD simulations exploring homogeneous catalytic reactions in solutions.
Acknowledgement: AS thanks OTKA for Grant K101115. NN is ${ }_{60}$ thankful to CSIR, India, for financial support. AL and GU thank Spanish MINECO (CTQ2011-23336, and RES-BSC). Our enthusiastic students A. Comas-Vives, G. Kovács, J. Daru are also acknowledged.

\section{${ }_{65}$ Notes and references}

${ }^{a}$ Chemical Research Center of HAS, Pusztaszeri fflt 59-67, 1025 Budapest (Hungary), Fax: (+36) 13257554, E-mail: stirling@chemres.hu ${ }^{b}$ Department of Chemistry, Indian Institute of Technology Kanpur, Kanpur, 208016 (India), Fax: (+91) 512-259-7436, 70 E-mail:nnair@iitk.ac.in

${ }^{b}$ Departament de Química, Universitat Autònoma de Barcelona, 08193 Bellaterra, Catalonia (Spain), Fax: (+34) 935812477

E-mail:agusti@klingon.uab.es,gregori@klingon.uab.es

$\dagger$ Electronic Supplementary Information (ESI) available: [details of any 75 supplementary information available should be included here]. See DOI: $10.1039 / \mathrm{b} 000000 \mathrm{x} /$

1 J. Smidt, W. Hafner, R. Jira, R. Sieber, J. Sedlmeier, A. Saber, Angew. Chem., 1962, 74, 93.

R. Jira, Angew. Chem. Int. Ed., 2009, 48, 9034.

J.A. Keith, P.M. Henry, Angew. Chem. Int. Ed., 2009, 48, 9038.

R.I. McDonald, G. Liu, S.S. Stahl, Chem. Rev., 2011 111, 2981

J. Tsuji, H. Nagashima, H. Nemoto, Org. Synth., 1984, 62, 9.

K.M.Gligorich, M.S. Sigman, Chem. Commun., 2009, 3854.

P.M. Henry, J. Am. Chem. Soc., 1964, 86, 3246.

N. Gregor, K. Zaw, P.M. Henry, Organometallics, 1984, 3, 1251.

9 J. Smidt, W. Hafner, R. Jira, R. Sieber, S. Sedlmeier, A. Sabel, Angew. Chem. Int. Ed. Eng., 1962, 1, 80.

10 P.M Henry, J. Org. Chem. 1973, 38, 2415.

11 D.J. Nelson, R. Li, C. Brammer, J. Chem. Am. Soc., 2001, 123, 1564.

12 J.K. Stille, R. Divakaruni, J. Am. Chem. Soc., 1978, 100, 1303.

T. Majima, H. Kurosawa, J. Chem. Soc. Chem. Commun., 1977, 610.

14 B. Akermark, B.C. Söderberg, S.S. Hall, Organometallics, 1987, 6, 2608.

15 J.-E. Bäckvall, B. Akermark, S.O. Ljunggren, J. Am. Chem. Soc., 1979, 101, 2411

16 R. Jira, in Applied Homogeneous Catalysis with Organomeetallic Compounds, Eds. B. Cornils, W.A. Herrman, Wiley-VCH, Weinheim, 2002, p. 386.

17 J.W. Francis, P.M. Henry, J. Mol. Cat. A, 1995, 99, 77.

18 W.K. Wan, K. Zaw, P.M. Henry, Organometallics, 1988, 7, 1677.

19 J.W. Francis, P.M. Henry, Organometallics, 1991, 10, 3498.

J.W. Francis, P.M. Henry, Organometallics, 1992, 11, 2832.

21 O. Hamed, P.M. Henry, C. Thompson, J. Org. Chem., 1999, 64, 774.

22 T. Hosokawa, T. Nomura, S.-I. Murahashi, J. Organomet. Chem., 1998, 551, 387.

23 B.J. Anderson, J.A. Keith, M.S. Sigman, J. Am. Chem. Soc., 2010, 132, 11872.

24 F. Jensen, Introduction to Computational Chemistry, Wiley, 2007.

25 H. B. Schlegel, WIREs Comput. Mol. Sci., 2011, 1, 790.

26 Y. Minenkov, A. Singstad, G. Occhipinti, V. R. Jensen, Dalton Trans., 2012, 41, 5526.

27 M. Steinmetz, S. Grimme, ChemistryOpen, 2013, 2, 115.

28 A. Fernandez-Ramos, J. A. Miller, S. J. Klippenstein, D. G. Truhlar, Chem. Rev., 2006, 106, 4518.

29 K. Fukui, Acc. Chem. Res., 1981, 14, 363.

30 J. Tomasi, B. Mennucci, R. Cammi, Chem. Rev. 2005, 105, 2999.

31 O. Eisenstein, R. Hoffmann, J. Am. Chem. Soc., 1981, 103, 4308.

32 J.-E. Bäckvall, E. E. Bjorkman, L. Pettersson, P. Siegbahn, J. Am. Chem. Soc., 1984, 106, 4369.

33 H. Fujimoto, T. Yamasaki, J. Am. Chem. Soc., 1986, 108, 578.

34 P. E. M. Siegbahn, J. Am. Chem. Soc., 1995, 117, 5409. 
35 P. E. M. Siegbahn, J. Phys. Chem., 1996, 100, 14672.

36 S. A. Beyramabadi, H. Eshtiagh-Hosseini, M. R. Housaindokht, A. Morsali, Organometallics, 2008, 27, 72.

37 J.A. Keith, R.J. Nielsen, J. Oxgaard, W.A. Goddard, P.M. Henry Organometallics, 2009, 28, 1618.

38 S. A. Beyramabadi, H. Eshtiagh-Hosseini, M. R. Housaindokht, A. Morsali, J. Mol. Struc. THEOCHEM , 2009, 903, 108.

39 J. A. Keith, J. Oxgaard, W. A. Goddard, J. Am. Chem. Soc., 2006, 128, 3132.

40 D. Kragten, R. A. van Santen, J.J. Lerou, J. Phys. Chem. A, 1999, 103,80 .

41 J. A. Keith, R. J. Nielsen, J. Oxgaard, W. A. Goddard, J. Am. Chem. Soc., 2007, 129, 12342.

42 D. Marx, J. Hutter, Ab Initio Molecular Dynamics: Basic Theory and Advanced Methods, Cambridge University Press: Cambridge, U. K., 2009.

43 A. Barducci, M. Bonomi, M. Parrinello, WIREs Comput. Mol. Sci. 2011, 1, 826.

44 N. N. Nair, J. Phys. Chem. B, 2011, 115, 2312.

45 V. Imandi, S. Kunnikuruvan, and N. N. Nair, Chem. Eur. J., 2013, 19, 4724 .

46 G. Kovács, A. Stirling, A. Lledós, G. Ujaque, Chem. Eur. J., 2012, 18, 5612 .

47 J. J. Cruywagen, R. J. Kriek, J. Coord. Chem., 2007, 60, 439;

48 A. Comas-Vives, A. Stirling, A. Lledós, G. Ujaque, Chem. Eur. J., 2010, 16, 8738

49 R. F. Heck, Hercules Chem., 1968, 57, 12. 